\title{
Broad-band properties of the hard X-ray cataclysmic variables IGR J00234+6141 and 1RXS J213344.1+510725^
}

\author{
G. Anzolin ${ }^{1,2}$, D. de Martino ${ }^{2}$, M. Falanga ${ }^{3}$, K. Mukai ${ }^{4}$, J.-M. Bonnet-Bidaud ${ }^{3}$, M. Mouchet ${ }^{5}$, \\ Y. Terada ${ }^{6}$, and M. Ishida ${ }^{7}$
}

1 Dipartimento di Astronomia, Università di Padova, vicolo dell'Osservatorio 3, 35122 Padova, Italy e-mail: gabriele.anzolin@unipd.it

2 INAF - Osservatorio Astronomico di Capodimonte, salita Moiariello 16, 80131 Napoli, Italy e-mail: demartino@oacn.inaf.it

3 CEA Saclay, DSM/DAPNIA/Service d'Astrophysique, 91191 Gif-sur-Yvette, France e-mail: [mfalanga; bonnetbidaud]@cea.fr

${ }^{4}$ CRESST and X-Ray Astrophysics Laboratory, NASA Goddard Space Flight Center, Greenbelt, MD 20771, USA and Department of Physics, University of Maryland, Baltimore County, 1000 Hilltop Circle, Baltimore, MD 21250, USA e-mail: koji.mukai@nasa.gov

5 Laboratoire APC, Université Denis Diderot, 10 rue Alice Domon et Léonie Duquet, 75005 Paris, France and LUTH, Observatoire de Paris, Section de Meudon, 5 place Jules Janssen, 92195 Meudon, France e-mail: martine.mouchet@obspm. fr

6 Department of physics, Saitama University, Saitama, Japan e-mail: terada@phy.saitama-u.ac.jp

7 Institute of Space and Astronautical Science, 3-1-1, Yoshinodai, Sagamihara, Kanagawa 229-8150, Japan e-mail: ishida@astro.isas.jaxa.jp

Received 9 February 2009 / Accepted 25 April 2009

\section{ABSTRACT}

\begin{abstract}
Context. A significant number of cataclysmic variables have been detected as hard X-ray sources in the INTEGRAL survey, most of them of the magnetic intermediate polar type.

Aims. We present a detailed X-ray broad-band study of two new sources, IGR J00234+6141 and 1RXS J213344.1+510725, that allow us to classify them as secure members of the intermediate polar class.

Methods. Timing and spectral analysis of IGR J00234+6141 are based on an XMM-Newton observation and INTEGRAL publicly available data. For 1RXS J213344.1+510725, we use XMM-Newton and Suzaku observations at different epochs, as well as INTEGRAL publicly available data.

Results. We determine a spin period of $561.64 \pm 0.56 \mathrm{~s}$ for the white dwarf in IGR J00234+6141. The X-ray pulses are observed up to $\sim 2 \mathrm{keV}$. From XMM-Newton and Suzaku observations of 1RXS J213344.1+510725, we find a rotational period of $570.862 \pm$ $0.034 \mathrm{~s}$. The observations span three epochs where the pulsation is observed to change at different energies both in amplitude and shape. In both objects, the spectral analysis spanned over a wide energy range, from 0.3 to $100 \mathrm{keV}$, shows the presence of multiple emission components absorbed by dense material. The X-ray spectrum of IGR J00234+6141 is consistent with a multi-temperature plasma with a maximum temperature of $\sim 50 \mathrm{keV}$. In 1RXS J213344.1+510725, multiple optically thin components are inferred, as well as an optically thick (blackbody) soft X-ray emission with a temperature of $\sim 100 \mathrm{eV}$. This adds 1 RXS J213344.1+510725 to the growing group of soft X-ray intermediate polars.

Conclusions. Though showing similar rotational periods and being hard X-ray sources, these two intermediate polars appear different in several respects, among which the presence of a soft X-ray component in 1RXS J213344.1+510725. This source also emits circularly polarized light in the optical band, thus joining the group of soft and polarized intermediate polars as a fifth member. How the hard X-ray and polarized emission are thermalized in these systems is a timely question.
\end{abstract}

Key words. stars: binaries: close - stars: individual: IGR J00234+6141 - stars: individual: 1RXS J213344.1+510725 stars: novae, cataclysmic variables - X-rays: stars - accretion, accretion disks

\section{Introduction}

The first deep survey above $20 \mathrm{keV}$ performed by the INTEGRAL satellite has allowed the detection of more than

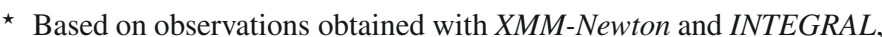
ESA science missions with instruments and contributions directly funded by ESA Member States and NASA, and with Suzaku, a Japan's mission developed at the Institute of Space and Astronautical Science of Japan Aerospace Exploration Agency in collaboration with US (NASA/GSFC, MIT) and Japanese institutions.
}

400 X-ray sources (Bird et al. 2007). The extensive survey of the Galactic plane has revealed that the contribution of Galactic $\mathrm{X}$-ray binaries, especially the cataclysmic variable (CV) type, is non-negligible in hard X-rays (Sazonov et al. 2006). Most of the hard X-ray emitting CVs were found to be magnetic intermediate polars (IPs) (Barlow et al. 2006). Up to now, IPs represent $\sim 5 \%$ of the INTEGRAL sources detected at that energy range and this number is likely to increase in the near future, as demonstrated by systematic optical follow-ups for some of the $\sim 100$ INTEGRAL unidentified sources (see e.g. 
Masetti et al. 2008). From the detection of thousands of discrete low-luminosity X-ray sources in a deep Chandra survey of regions close to the Galactic center, Muno et al. (2004) proposed that magnetic CVs of the IP type may represent a significant fraction of the Galactic background. Further support that magnetic CVs could still be a hidden population of faint X-ray sources and, therefore, play an important role in the X-ray emission of the Galaxy comes from recent studies of the Galactic ridge with INTEGRAL and RXTE (Revnivtsev et al. 2008).

IPs are believed to harbor weakly magnetized ( $B \lesssim 10 \mathrm{MG})$ white dwarfs (WDs) because of their fast asynchronous rotation with respect to the orbital period and of the lack of significant polarized emission in the optical/near-IR in most systems. This contrasts with the other group of magnetic CVs, the polars, that instead possess strongly magnetized ( $B \sim 10-230 \mathrm{MG}$ ) WDs rotating synchronously with the binary period. The X-ray properties of magnetic CVs are strictly related to the accretion mechanism onto the WD primary. Material from the late type companion is driven by the magnetic field lines onto the magnetic polar caps, where a shock develops (Aizu 1973) below which hard $\mathrm{X}$-rays and cyclotron radiation are emitted. Bremsstrahlung radiation is believed to be the dominant cooling mechanism in IPs (Wu et al. 1994), while cyclotron radiation may dominate in polars. The complex interplay between the two mechanisms greatly depends on both magnetic field strength and local mass accretion rates (Woelk \& Beuermann 1996; Fischer \& Beuermann 2001). Hence, if IPs indeed host weakly magnetized WDs with respect to polars, this could qualitatively explain why they are hard X-ray sources. The detection of a soft X-ray optically thick component in an increasing number of systems (Anzolin et al. 2008) poses further questions in the interpretation of the X-ray emission properties of IPs.

In the framework of an ongoing optical identification program, we identified two new members of the IP group: 1RXS J213344.1+510725 = IGR J21335+5105 (hereafter RX J2133) (Bonnet-Bidaud et al. 2006) and IGR J00234+6141 = 1RXS J002258.3+614111 (hereafter IGR 0023) (Bonnet-Bidaud et al. 2007). Both of them are hard CVs in the INTEGRAL source catalog (Bird et al. 2007).

The weak hard X-ray source IGR 0023 was detected by the INTEGRAL satellite during an observation of the Cassiopeia region of the Galaxy (den Hartog et al. 2006). The optical counterpart of IGR 0023 was identified by Masetti et al. (2006), who proposed a possible magnetic nature of this CV. A tentative $\sim 570$ s optical periodicity was recognized in the $R$ band (Bikmaev et al. 2006). However, a clear periodic modulation of $563.53 \pm 0.62 \mathrm{~s}$ was discovered with optical photometric data by Bonnet-Bidaud et al. (2007) and readily ascribed to the rotational period of the WD, while an orbital period of $4.033 \pm 0.005 \mathrm{~h}$ was derived from optical spectroscopy. The properties of the INTEGRAL spectrum, which is well fitted by a bremsstrahlung with a temperature of $31 \mathrm{keV}$, strongly support the magnetic nature of IGR 0023.

RX J2133 was identified as a hard X-ray point source from the ROSAT Galactic Plane Survey (Motch et al. 1998). A clear persistent optical light pulsation at $570.823 \pm 0.013 \mathrm{~s}$ was then discovered with fast optical photometry, while optical spectroscopy revealed an additional periodic variability at $7.193 \pm$ $0.016 \mathrm{~h}$ (Bonnet-Bidaud et al. 2006). These two periodicities were respectively identified as the WD spin and the orbital periods, thus suggesting RX J2133 as a member of the IP class with a relatively long orbital period which falls into the so-called IP gap between 6.5 and 9.5 h (Schenker et al. 2004). Katajainen et al. (2007) found also that RX J2133 emits optical circularly polarized light up to $\sim 3 \%$ and proposed that the WD magnetic field could be as high as $25 \mathrm{MG}$, one of the highest amongst IPs.

The X-ray variability and broad-band spectra of these two systems have been investigated using pointed XMM-Newton (Jansen et al. 2001) observations and publicly available hard X-ray data obtained with the INTEGRAL satellite (Winkler et al. 2003). In the case of RX J2133, we also present the temporal and spectral analysis of a pointed Suzaku (Mitsuda et al. 2007) observation.

\section{Observations and data reduction}

The summary of the observations of IGR 0023 and RX J2133 is reported in Table 1.

\subsection{The XMM-Newton observations}

For all our XMM-Newton observations, we reprocessed and analyzed the EPIC-pn (Strüder et al. 2001), MOS (Turner et al. 2001), RGS (den Herder et al. 2001) and OM (Mason et al. 2001) data using the standard reduction pipelines included in SAS 8.0 and the latest calibration files. For IGR 0023, because of a problem with the standard source detection task, the OM$U V M 2$ data were reprocessed at MSSL using an unreleased reduction routine. Heliocentric corrections were applied to the EPIC and OM data of both sources. The SAS tasks rmfgen and arfgen were used to generate the photon redistribution matrix and the ancillary region files for all the EPIC cameras and RGS instruments.

IGR 0023 was observed on July 10, 2007 (OBSID: 0501230201) with the EPIC-pn and MOS cameras operated in full frame imaging mode with the thin and medium filters, respectively. The total exposure times were $25 \mathrm{ks}$ for EPIC-pn and $26.6 \mathrm{ks}$ for both the MOS cameras. The RGS was operated in spectroscopy mode for a total exposure time of $26.9 \mathrm{ks}$. The OM was operated in fast imaging mode using sequentially the $V$ (5000-6000 $)$ and UVM2 (2000-2800 $\AA$ ) filters, for $9.8 \mathrm{ks}$ each.

A $28^{\prime \prime}$ aperture radius was used to extract EPIC light curves and spectra from a circular region centered on the source and from a background region located on the same CCD chip where the source was imaged. In order to improve the $\mathrm{S} / \mathrm{N}$ ratio, we filtered the data by selecting pattern pixel events up to double with zero quality flag for the EPIC-pn data, and up to quadruple pixel events for the EPIC-MOS data. The average background level of the EPIC cameras was quite low for almost all the duration of the observation, with the exception of a moderate flaring activity, which occurred during the last $\sim 2000$ s of the EPIC-pn exposure. This flare did not significantly affect the data used for the timing analysis, however we conservatively did not consider these events in the spectral analysis.

Due to the weakness of IGR 0023, the RGS data had poor $\mathrm{S} / \mathrm{N}$ ratios and therefore were not useful for spectral analysis.

Background subtracted OM- $V$ light curves were obtained with a binning time of $20 \mathrm{~s}$, while the OM-UVM2 light curves were provided by MSSL with a binning time of $10 \mathrm{~s}$. The average count rates were 3.00 counts $\mathrm{s}^{-1}$ in the $V$ band and 0.18 counts $\mathrm{s}^{-1}$ in the $U V M 2$ band, corresponding to instrumental magnitudes $V=16.8$ and $U V M 2=17.6$ and average fluxes of $7.4 \times 10^{-16} \mathrm{erg} \mathrm{cm}^{-2} \mathrm{~s}^{-1} \AA^{-1}$ and $4.0 \times$ $10^{-16} \mathrm{erg} \mathrm{cm}^{-2} \mathrm{~s}^{-1} \AA^{-1}$, respectively. As a comparison, the continuum flux of the optical spectrum obtained by Masetti et al. (2006) was $\sim 6 \times 10^{-16} \mathrm{erg} \mathrm{cm}^{-2} \mathrm{~s}^{-1} \AA^{-1}$. 
Table 1. Summary of the X-ray observations of IGR 0023 and RX J2133.

\begin{tabular}{|c|c|c|c|c|c|}
\hline Object & Instrument & Date & UT (start) & Exposure time $(\mathrm{s})^{a}$ & Net count rate $\left(\right.$ counts s $\left.{ }^{-1}\right)$ \\
\hline \multirow[t]{14}{*}{ IGR 0023} & EPIC-pn & $2007-07-10$ & $05: 58$ & 24660 & $0.984 \pm 0.007$ \\
\hline & EPIC-MOS & & 05:36 & 26540 & $0.345 \pm 0.004$ \\
\hline & RGS & & $05: 35$ & 26706 & $0.038 \pm 0.001$ \\
\hline & OM-V & & $05: 44$ & 1960 & $3.26 \pm 0.04$ \\
\hline & & & $06: 22$ & 1959 & $3.12 \pm 0.04$ \\
\hline & & & 07:01 & 1960 & $2.79 \pm 0.04$ \\
\hline & & & 08:12 & 1962 & $2.97 \pm 0.04$ \\
\hline & & & $08: 50$ & 1959 & $2.85 \pm 0.04$ \\
\hline & OM-UVM2 & & 09:28 & 1960 & $0.16 \pm 0.01$ \\
\hline & & & 10:06 & 1959 & $0.19 \pm 0.01$ \\
\hline & & & $10: 45$ & 1960 & $0.20 \pm 0.01$ \\
\hline & & & $11: 23$ & 1960 & $0.18 \pm 0.01$ \\
\hline & & & $12: 01$ & 1960 & $0.18 \pm 0.01$ \\
\hline & IBIS/ISGRI & & & $\sim 6900000$ & $0.10 \pm 0.01$ \\
\hline \multirow[t]{25}{*}{ RX J2133 } & EPIC-pn & $2005-05-29$ & $13: 33$ & 13585 & $5.42 \pm 0.02$ \\
\hline & EPIC-MOS & & $12: 47$ & 16580 & $1.398 \pm 0.008$ \\
\hline & RGS & & $13: 28$ & 13890 & $0.147 \pm 0.004$ \\
\hline & OM-B & & 10:11 & 2320 & $24.9 \pm 0.1$ \\
\hline & & & $10: 55$ & 2319 & $23.2 \pm 0.1$ \\
\hline & & & $11: 39$ & 2319 & $22.4 \pm 0.1$ \\
\hline & & & $12: 23$ & 2319 & $22.1 \pm 0.1$ \\
\hline & & & 13:07 & 2320 & $23.7 \pm 0.1$ \\
\hline & OM-UVM2 & & $13: 51$ & 2319 & $0.69 \pm 0.02$ \\
\hline & & & $14: 35$ & 2321 & $0.68 \pm 0.02$ \\
\hline & & & $15: 20$ & 2320 & $0.89 \pm 0.02$ \\
\hline & & & $16: 03$ & 2320 & $0.85 \pm 0.02$ \\
\hline & & & $16: 48$ & 2319 & $0.87 \pm 0.02$ \\
\hline & EPIC-pn & 2005-07-06 & $18: 03$ & 9871 & $5.12 \pm 0.03$ \\
\hline & EPIC-MOS & & $17: 05$ & 13680 & $1.105 \pm 0.009$ \\
\hline & RGS & & $17: 04$ & 13910 & $0.120 \pm 0.005$ \\
\hline & OM-UVM2 & & $17: 31$ & 1681 & $0.60 \pm 0.02$ \\
\hline & & & $18: 16$ & 1679 & $0.70 \pm 0.02$ \\
\hline & & & $19: 20$ & 1680 & $0.64 \pm 0.02$ \\
\hline & & & $19: 53$ & 1680 & $0.69 \pm 0.02$ \\
\hline & & & $20: 27$ & 1679 & $0.59 \pm 0.02$ \\
\hline & IBIS/ISGRI & & & $\sim 3740000$ & $0.55 \pm 0.02$ \\
\hline & XIS-FI & 2006-04-29 & $06: 50$ & 84288 & $0.738 \pm 0.002$ \\
\hline & XIS-BI & & $06: 50$ & 84288 & $0.892 \pm 0.004$ \\
\hline & HXD & & $06: 50$ & 62879 & $0.739 \pm 0.004$ \\
\hline
\end{tabular}

${ }^{a}$ We report total exposure times for the INTEGRAL observations and net exposure times for the others.

RX J2133 was observed on May 29, 2005 (OBSID: 0302100101) with all the EPIC cameras operated in full frame mode and with the medium filter. Due to high background radiation the observation time was shortened and the net exposure times were 13.6 ks for EPIC-pn, $16.5 \mathrm{ks}$ for EPIC-MOS and $13.9 \mathrm{ks}$ for RGS, the latter operated in spectroscopy mode. The $\mathrm{OM}$, operated in fast imaging mode, was used sequentially with the $B$ filter, covering the spectral range 3900-4900 $\AA$, and the UVM2 filter for $11.6 \mathrm{ks}$ each. RX $\mathrm{J} 2133$ was observed again on July 06, 2005 (OBSID: 0302100301) with the same instrumental configurations and with net exposure times of $9.9 \mathrm{ks}$ for EPIC-pn, $13.7 \mathrm{ks}$ for EPIC-MOS and $13.9 \mathrm{ks}$ for RGS. The OM was only operated with the $U V M 2$ filter for a total exposure time of $8.4 \mathrm{ks}$. The source was found at about the same count rate at the two epochs in all instruments.

EPIC light curves and spectra of source and background were extracted from circular regions of $37^{\prime \prime}$ radius. The same filtering adopted for IGR 0023 was applied to improve the $\mathrm{S} / \mathrm{N}$ ratio for both cameras. During the observation of May 2005 the background was moderately active, but not enough to affect the timing analysis. Filtering of higher background periods was done only during the extraction of spectra from both EPIC and
RGS data. These spectra were rebinned to have a minimum of 25 and 20 counts per bin, respectively.

OM $B$ and $U V M 2$ light curves were extracted with a binning time of $10 \mathrm{~s}$ and $20 \mathrm{~s}$, respectively. The average count rates were about 25 counts s$^{-1}$ in the $B$ band and 0.7 counts s ${ }^{-1}$ in the UVM2 band, corresponding to instrumental magnitudes $B=15.8$ and $U V M 2=16.2$. These translate into $B$ and $U V M 2$ fluxes of $3 \times 10^{-15} \mathrm{erg} \mathrm{cm}^{-2} \mathrm{~s}^{-1} \AA^{-1}$ and $1.5 \times$ $10^{-15} \mathrm{erg} \mathrm{cm}^{-2} \mathrm{~s}^{-1} \AA^{-1}$, respectively.

\subsection{The INTEGRAL observations}

The INTEGRAL IBIS/ISGRI (Ubertini et al. 2003; Lebrun et al. 2003) hard X-ray data of both sources were extracted from all pointings within $12^{\circ}$ from the source positions, spanning from March 2003 to October 2006. The total effective exposure times are $\sim 6.9 \mathrm{Ms}$ ( 2875 pointings) and $\sim 3.74 \mathrm{Ms}$ (1563 pointings) for IGR 0023 and RX J2133, respectively. To study the weak persistent X-ray emission, the time averaged ISGRI spectra have been obtained from mosaic images in five energy bands, logarithmic spaced between 20 and $100 \mathrm{keV}$. Data were reduced with the 
standard OSA software version 7.0 and, then, analyzed using the algorithms described by Goldwurm et al. (2003).

\subsection{The Suzaku observations of RX J2133}

RX J2133 was observed with Suzaku between Apr. 29, 2006 and May 1, 2006 (sequence number 401038010). We have analyzed data from the X-ray imaging spectrometer (XIS, Koyama et al. 2007) and the non-imaging hard X-ray detector (HXD, Takahashi et al. 2007). The observation was done with the object at the "HXD-nominal" pointing position, $\sim 5$ ' off-axis from the center of field-of-view (FOV) of the XIS, to optimize the $\mathrm{S} / \mathrm{N}$ ratio of the HXD data. We based our analysis on data processed using the V2.0.6.13 pipeline released as a part of HEADAS 6.3.1.

For the XIS, we updated the energy scale calibration using the February 1, 2008 release of the calibration database. We then applied the following screening criteria: attitude control system in nominal mode, pointing within 1.5 of the mean direction, XIS data rate medium or high, the satellite outside the South Atlantic Anomaly (SAA) and at least $180 \mathrm{~s}$ after the last SAA passage, elevation above Earth limb $>5^{\circ}$, elevation about the bright Earth limb $>15^{\circ}$. An inspection of the XIS image revealed a second source, near the center of the FOV, with a flux of $6.7 \times$ $10^{-13} \mathrm{erg} \mathrm{cm}^{-2} \mathrm{~s}^{-1}$ in the $2-10 \mathrm{keV}$ band. This same source is seen in the XMM-Newton data at similar flux levels ${ }^{1}$. Although being faint, we conservatively excluded this source from our analysis of the XIS data. We cannot do so for the HXD data analysis, but it is expected to have a negligible effect.

We used a 3.5 radius extraction region and an annular extraction region of 7'.5 outer and $4^{\prime}$ inner radii for the background, both centered on the position of RX J2133. For spectroscopy, we summed the data and the responses of the three XIS-FI units because they have nearly identical responses. For photometry, we added background subtracted light curves from all the XIS units over the energy range $0.3(\mathrm{FI}) / 0.2(\mathrm{BI})-12 \mathrm{keV}$.

For the HXD data, we took the PIN event data from the processing pipeline and applied the dead time correction. We obtained the "tuned" non X-ray background files (Fukazawa et al. 2009), estimated by the HXD team using LCFITDT method. For phase-resolved spectroscopy, we used the phase-averaged background and dead-time fraction, since both tend to vary on a longer time scale.

\section{Data analysis and results}

\subsection{IGR 0023}

\subsubsection{The X-ray variability}

The EPIC-pn light curve extracted in the energy range $0.2-10.0 \mathrm{keV}$ and binned in $20 \mathrm{~s}$ time intervals reveals a clear variability with a time scale of the order of $\sim 10 \mathrm{~min}$. We have not analyzed the EPIC-MOS light curves because they were too noisy to provide reliable results. The power spectrum of the fullband EPIC-pn light curve (see Fig. 1) shows significant peaks at $\omega \sim 154 \mathrm{~d}^{-1}$, at $2 \omega$ and also at low frequencies. A peak at $f_{1} \sim 22 \mathrm{~d}^{-1}$, although its significance is below $2 \sigma$, is close to a pseudo-periodicity detected in the optical (Bonnet-Bidaud et al. 2007).

A sinusoidal fit was performed on the EPIC-pn light curve previously corrected for low frequency trends by using a third-order polynomial. We used three sinusoids with different

\footnotetext{
1 We have not investigated the nature of this second source, but a likely explanation is that it is a background AGN.
}

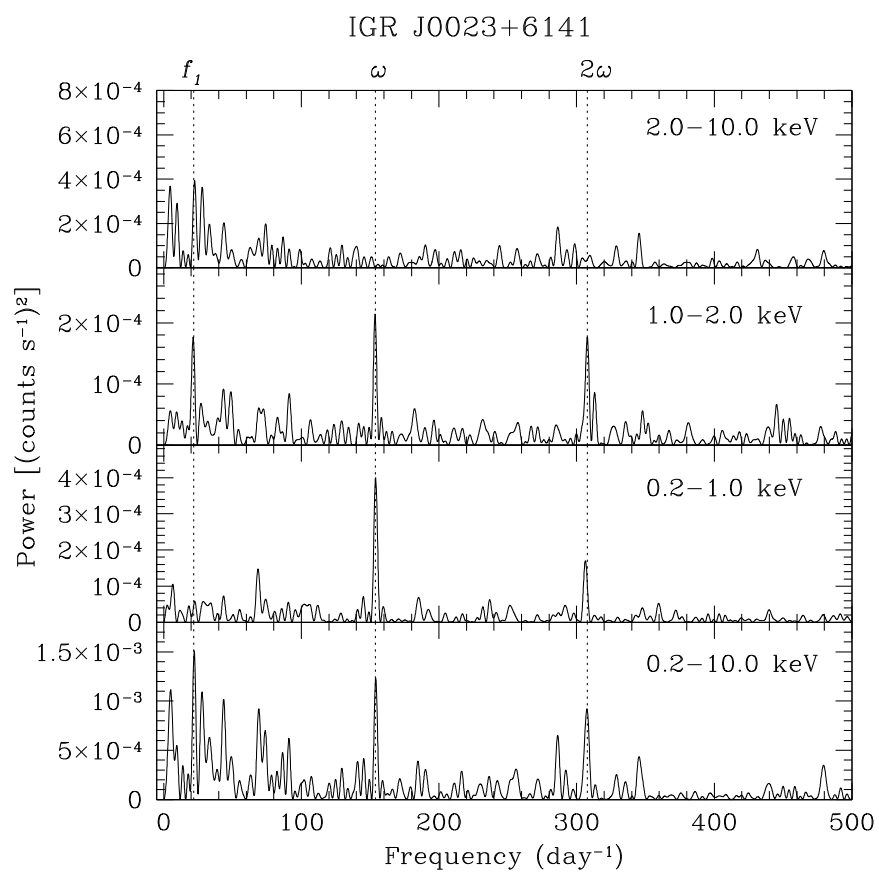

Fig. 1. Power spectra of the EPIC-pn data of IGR 0023 in selected energy ranges. From bottom to top: $0.2-10.0 \mathrm{keV}, 0.2-1.0 \mathrm{keV}$, 1.0-2.0 keV and 2.0-10.0 keV. Vertical dotted line indicate the frequencies $\omega, 2 \omega$ and $f_{1}$ (see text).

frequencies accounting for all the observed peaks, thus finding $\omega=153.83 \pm 0.15 \mathrm{~d}^{-1}$ and $f_{1}=21.94 \pm 0.15 \mathrm{~d}^{-1}$ (errors are at the $1 \sigma$ confidence level). The inferred period $P_{\omega}=561.64 \pm$ 0.56 s can be identified with the WD spin period, since the difference with the optical period of Bonnet-Bidaud et al. (2007) is not significant.

A Fourier analysis was also performed on EPIC-pn light curves extracted with a $40 \mathrm{~s}$ binning time in selected energy bands: $0.2-1.0 \mathrm{keV}, 1.0-2.0 \mathrm{keV}$ and $2.0-10.0 \mathrm{keV}$ (see Fig. 1). Peaks at $\omega$ and $2 \omega$ are clearly detected in the low and intermediate energy bands, while they do not appear at high energies. Instead, the pseudo-periodicity at low frequency is visible only in the intermediate $1-2 \mathrm{keV}$ band.

We then folded the EPIC-pn light curves with the $561.64 \mathrm{~s}$ spin period (Fig. 2) using the time of maximum obtained from the sinusoidal fit of the $0.2-10.0 \mathrm{keV}$ light curve: HJD = $2454291.8667(2)$. These light curves show a quasi-sinusoidal modulation only below $2.0 \mathrm{keV}$, with a secondary maximum at $\phi \sim 0.5$. The pulse amplitude is $\sim 50 \%$ in the $0.2-1.0 \mathrm{keV}$ range and $\sim 16 \%$ in the $1.0-2.0 \mathrm{keV}$ range. The count ratio between the $1.0-2.0 \mathrm{keV}$ and the $0.2-1.0 \mathrm{keV}$ bands indicates an hardening of the emission at the secondary maximum.

\subsubsection{The visible and UV light curves}

The power spectrum of the OM- $V$ light curve shows a strong peak at the X-ray spin period, while that of the UVM2 light curve does not reveal any significant peak. The average count rate is not constant in the 5 observations with the $V$ filter spanning $\sim 3 / 4$ of the orbital cycle, probably suggesting a dependence on the orbital period. The spin-folded OM- $V$ light curve (lower panel of Fig. 2) is pretty sinusoidal, with an amplitude of $8 \pm 1 \%$, and shows a single peak at a phase consistent with that of the main maximum of the X-ray pulse. We notice that 


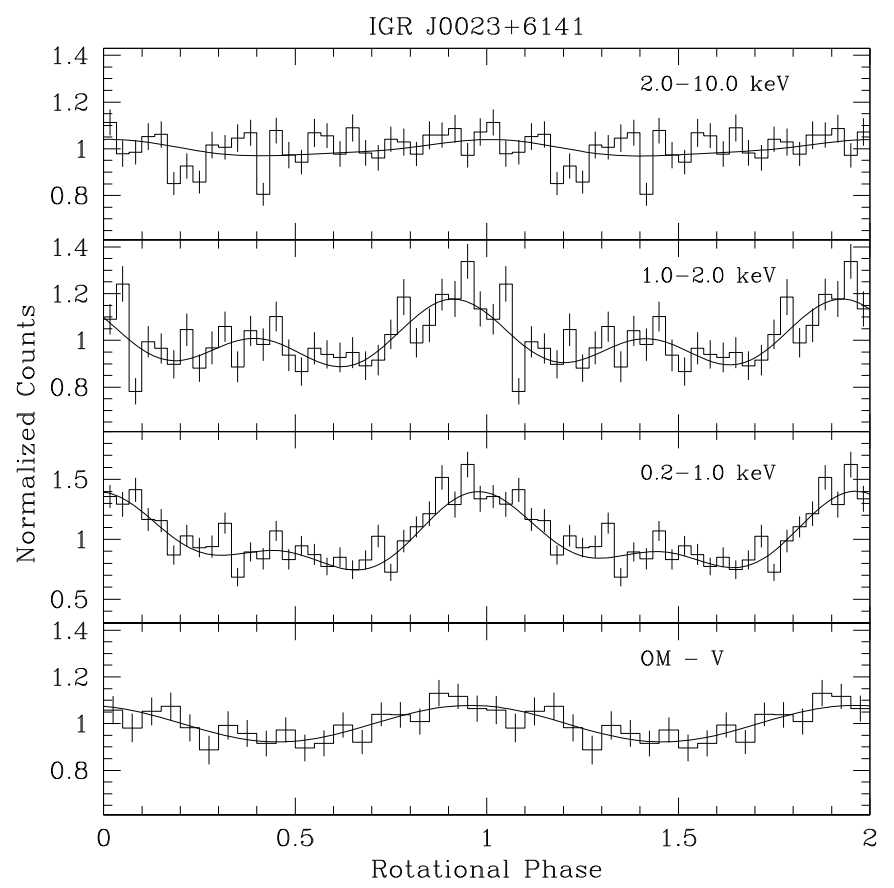

Fig. 2. The spin pulse of IGR 0023 in selected energy ranges of the EPIC-pn and in the OM- $V$ band. All the light curves are folded at the $561.6 \mathrm{~s} X$-ray spin period using the time of maximum quoted in the text. Solid lines represent sinusoidal fits to the corresponding data.

Bonnet-Bidaud et al. (2007) also found a single peaked pulsation in their observations carried out with the Gunn-g filter.

\subsubsection{Spectral properties of IGR 0023}

The EPIC-pn and combined MOS spectra (in the range $0.3-10.0 \mathrm{keV}$ ) and the IBIS/ISGRI spectrum (in the range 20-100 keV) were simultaneously analyzed with the XSPEC 12 package. An absorbed isothermal optically thin MEKAL component plus a zero width Gaussian at $6.4 \mathrm{keV}$ fit relatively well the spectrum $\left(\chi_{v}^{2}=1.04\right)$, but the temperature is unconstrained $(>78 \mathrm{keV})$. The fit improves using a multi-temperature CEMEKL emission component and a dense $\left(N_{\mathrm{H}} \sim 10^{23} \mathrm{~cm}^{-2}\right)$ absorber covering $\sim 40 \%$ of the source (model A in Table 4), although the $\alpha$ parameter assumes an unreasonably high value. Metal abundances are consistent, within errors, with the solar values. The total absorber is likely of interstellar origin, as it is comparable to that of the total Galactic absorption in the direction of the source $\left(N_{\mathrm{H}, \mathrm{gal}}=7.4 \times 10^{21} \mathrm{~cm}^{2}\right.$, Dickey \& Lockman (1990)). The dense partial absorber, instead, is likely located close to the source, as suggested by the energy dependence of the spin light curve. We also obtained similar quality fits by substituting the CEMEKL component with 2 (Fig. 3) or 3 MEKALs (model $\mathrm{B}$ and $\mathrm{C}$ in Table 4 , respectively). In both cases, we find a low $(k T \sim 0.17 \mathrm{keV})$ and an intermediate temperature $(k T \sim 10 \mathrm{keV})$ components, and, in model C, a lower limit to the temperature of the third MEKAL $k T>27 \mathrm{keV}$. The observed X-ray flux in the $0.2-10.0 \mathrm{keV}$ range is $6.8 \times$ $10^{-12} \mathrm{erg} \mathrm{cm}^{-2} \mathrm{~s}^{-1}$. We notice that only model $\mathrm{B}$ and model $\mathrm{C}$ account for the O VII (21.9 $\AA$ ) and O VIII (19.1 $\AA$ ) lines barely detectable in the low quality RGS spectra.

The INTEGRAL spectrum above $60 \mathrm{keV}$ seems to be underpredicted by the three best-fit models but, given the poor $\mathrm{S} / \mathrm{N}$ ratio, the excess of counts is not significant. The addition of a reflection component, suggested by the presence of the

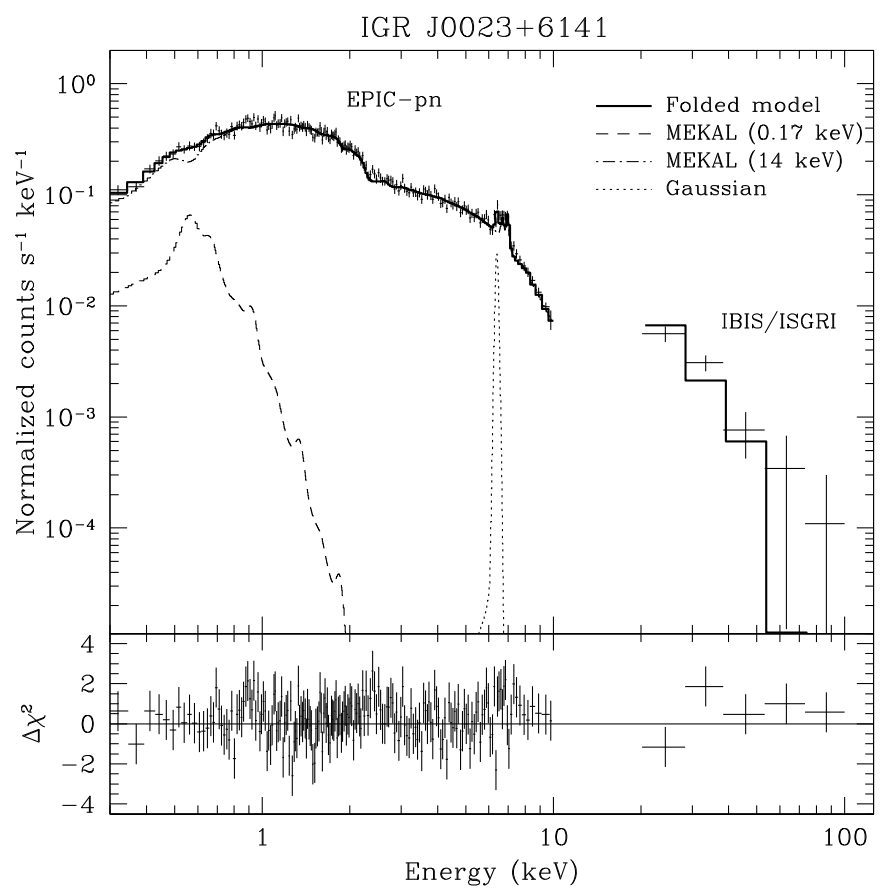

Fig. 3. The EPIC-pn and INTEGRAL spectra of IGR 0023 are shown with model B discussed in the text and parameters reported in Table 4 (we do not report the combined MOS spectrum for clarity). Each spectral component that contributes to the best-fit model is shown separately. The bottom panel shows the residuals expressed in terms of $\sigma$.

Table 2. Best fit parameters of the phase-resolved spectra of IGR 0023.

\begin{tabular}{lll}
\hline \hline Parameters & Maximum 1 & Maximum 2 \\
\hline$N_{\mathrm{H}}\left(10^{23} \mathrm{~cm}^{-2}\right)$ & $1.5_{-0.6}^{+1.0}$ & $1.2_{-0.3}^{+0.5}$ \\
Cov. Frac. & $0.36_{-0.07}^{+0.06}$ & $0.51 \pm 0.04$ \\
$C_{1}\left(10^{-4}\right)$ & $1.2_{-0.6}^{+0.3}$ & $0.5 \pm 0.5$ \\
$C_{2}\left(10^{-2}\right)$ & $1.5_{-0.1}^{+0.2}$ & $1.7 \pm 0.1$ \\
\hline$F_{0.2-10.0 \mathrm{keV}}$ & 2.06 & 2.00 \\
$\left(10^{-12} \mathrm{erg} \mathrm{cm} \mathrm{s}^{-1}\right)$ & & \\
\hline$\chi_{v}^{2}\left(\chi^{2} /\right.$ d.o.f. $)$ & $1.01(259 / 257)$ & $1.10(258 / 235)$ \\
\hline
\end{tabular}

Fe line at $6.4 \mathrm{keV}$, does not improve the fits. We also fitted the broad-band spectrum of IGR 0023 with the recent post-shock region (PSR) model of Suleimanov et al. (2008) that computes the emergent spectrum taking also into account the Compton scattering $\left(\chi^{2} /\right.$ d.o.f. $\left.=136 / 110\right)$. We obtained a shock temperature $k T_{\text {shock }}=51 \pm 11 \mathrm{keV}$ and absorption parameters consistent with those found with models $\mathrm{A}-\mathrm{C}$. The contribution of the Compton scattering is found to be $\sim 10 \%$ and, within errors, does not seem to affect the temperature determination.

A phase-resolved analysis of the EPIC-pn spectrum of IGR 0023 was performed selecting two phase intervals centered on the two maxima of the spin pulse $(\phi=0.85-1.15$ and $\phi=0.35-0.65$, respectively). We used model B, since model A and $\mathrm{C}$ would give badly constrained parameters. The hydrogen column density of the total absorber, the temperatures of the two MEKALs and the metal abundance were kept fixed to the values obtained for the average spectrum. As shown in Table 2, the normalization of the low-temperature optically thin component is significantly lower at the secondary maximum, where we also find a marginal evidence of an increase of the covering fraction of the local absorber. 
1RXS J2133.7+5107

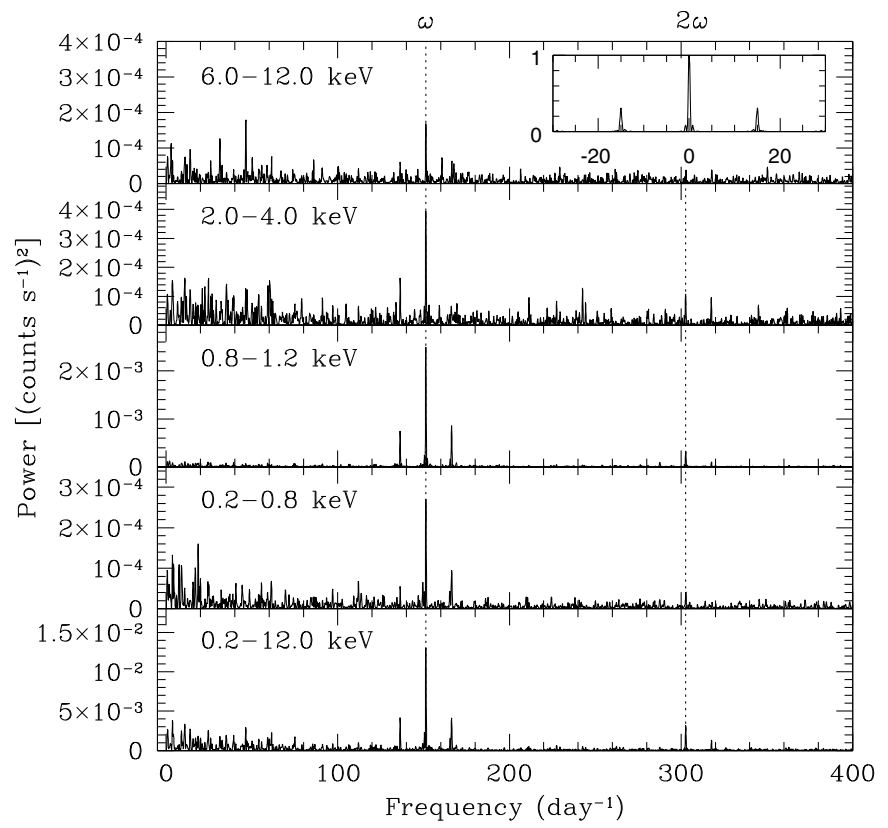

Fig. 4. Power spectra of the Suzaku XIS light curves of RX J2133 in selected energy ranges. From bottom to top: $0.2-12.0 \mathrm{keV}, 0.2-0.8 \mathrm{keV}$, $0.8-1.2 \mathrm{keV}, 2.0-4.0 \mathrm{keV}$ and $6.0-12.0 \mathrm{keV}$. Vertical dotted lines indicate the frequencies $\omega$ and $2 \omega$ (see text). The spectral window is shown in the inset.

\section{2. $R X J 2133$}

\subsubsection{The X-ray periodicities in $\mathrm{RX} \mathrm{J} 2133$}

The EPIC light curves, extracted in the $0.2-12.0 \mathrm{keV}$ energy range and binned in $5 \mathrm{~s}$ time intervals, and the Suzaku XIS light curve, extracted with a binning time of $8 \mathrm{~s}$, show a variability of the order of $10 \mathrm{~min}$. In the EPIC light curve of May 2005 a quasisinusoidal trend with an apparent period of $\sim 2 \mathrm{~h}$ is found, while in July $2005 \mathrm{a} \sim 1 \mathrm{~h}$ pseudo-periodicity is detected. However, in the later XIS observation we do not find any evidence of such a variability.

We then performed a Fourier analysis on the light curves at the three epochs. Peaks at the optically identified spin frequency, $\omega$, and at $2 \omega$ are clearly detected. In Fig. 4 we report the full-band XIS power spectra, as well as that obtained in different energy bands. Sinusoidal fits to the full-band light curves give $\omega=151.30 \pm 0.25 \mathrm{~d}^{-1}, \omega=151.55 \pm 0.78 \mathrm{~d}^{-1}$ and $\omega=151.350 \pm 0.009 \mathrm{~d}^{-1}$ for the May and July 2005 XMM-Newton and April 2006 Suzaku data sets, respectively. In the analysis of the EPIC-pn light curves, a third sinusoid has to be included, accounting for the low frequency variations, thus giving $f_{\text {May } 2005}=43.78 \pm 0.29 \mathrm{~d}^{-1}$ and $f_{\text {July } 2005}=27.87 \pm$ $0.41 \mathrm{~d}^{-1}$. We identify the precise $S u z a k u$ period $P_{\omega}=570.862 \pm$ $0.034 \mathrm{~s}$ with the true spin period of the accreting WD. This agrees, within errors, with the optical $570.823 \pm 0.013 \mathrm{~s}$ period.

We folded the EPIC-pn and XIS light curves at the $570.86 \mathrm{~s}$ $\mathrm{X}$-ray spin period using the time of maxima of the pulsation at $\omega$ : $\mathrm{HJD}_{\text {May } 2005}=2453520.1444(2)$, HJD July $2005=$ 2453 558.3134(4) and HJD April $2006=2453$ 856.09618(5). The three folded light curves (Fig. 5) present two maxima at phases 0.9 and 0.35 , with a dip at phase $\sim 0.1$ that is more evident in the May 2005 data. The full amplitude of the primary maximum at $\phi \sim 0.9$ is almost similar at the three epochs $(\sim 36 \%)$,

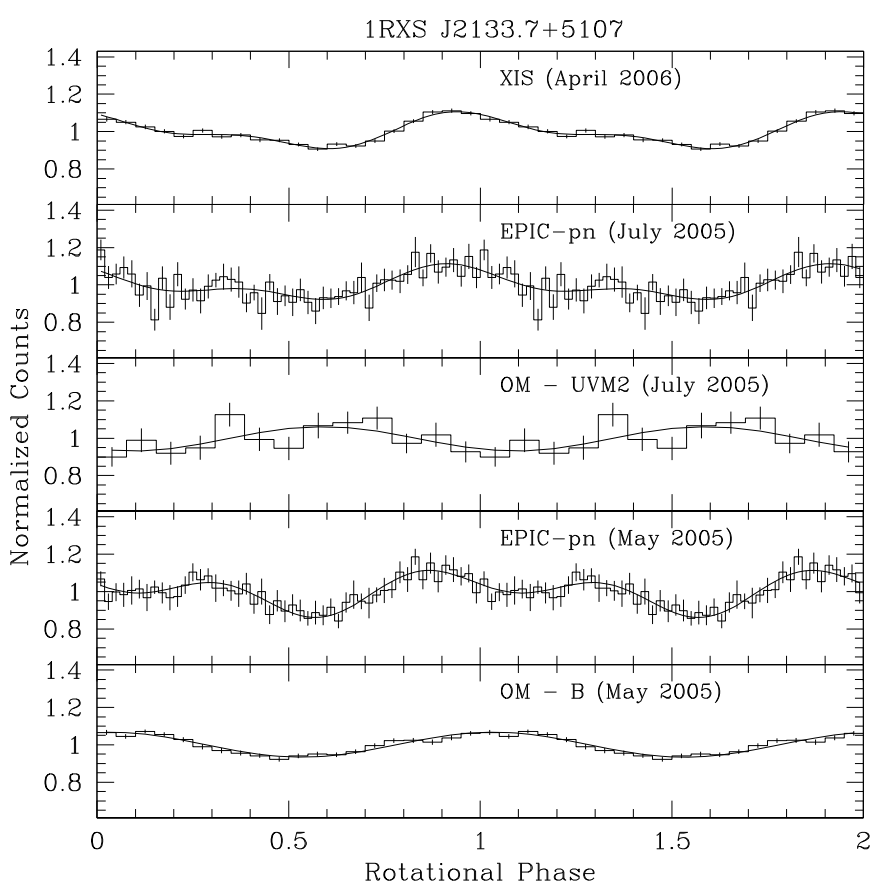

Fig. 5. Spin-folded light curves of RX J2133. From top to bottom: full-band XIS of April 2006, EPIC-pn and OM-UVM2 of July 2005, EPIC-pn and OM- $B$ of May 2005. Solid lines represent sinusoidal fits to the corresponding data.

while that of the secondary maximum decreases from $\sim 13 \%$ in May 2005 to $\sim 3 \%$ in 2006.

The energy-resolved EPIC-pn light curves (Fig. 6) generally show the secondary maximum dominating the emission in the soft $0.2-0.5 \mathrm{keV}$ band and the primary maximum dominating in the range $0.5-2.0 \mathrm{keV}$. At higher energies the two maxima have similar amplitudes of $\sim 10 \%$. The XIS light curves, though being broadly similar to the EPIC-pn light curves, present an evident secondary maximum only in the hard X-rays.

\subsubsection{The UV and optical light variability of $R X J 2133$}

The power spectrum of the OM- $B$ light curve shows a strong peak at the spin frequency (consistent within errors with the $\mathrm{X}$-ray and the previous optical determinations). We also detect power at low frequencies, but we cannot establish whether this indicates a true periodic variability. The OM- $B$ light curve of May 2005 folded at the spin period (see Fig. 5) is single peaked, with a broad maximum at phase $\sim 0$ and a full amplitude of $14 \%$. The UV light curve, instead, is almost unmodulated in May 2005, while in July 2005 it is sinusoidal with a maximum shifted by half a period with respect to the OM- $B$ light curve of May 2005. A similar antiphased behavior is also seen in some soft X-ray IPs, like UU Col (de Martino et al. 2006). However, we point out that each OM light curve covers only $\sim 41 \%$ of the orbital period and hence could be affected by orbital dependent changes of the spin modulation.

\subsubsection{The X-ray spectrum of $R X J 2133$}

The X-ray spectrum of RX J2133 was studied in the broad-band energy range $0.3-100 \mathrm{keV}$ using the EPIC-pn and combined MOS data at each epoch, together with the IBIS/ISGRI spectrum. We also analyzed the combined XIS-FI, XIS-BI and HXD spectra in the restricted range $0.3-40 \mathrm{keV}$, where the 


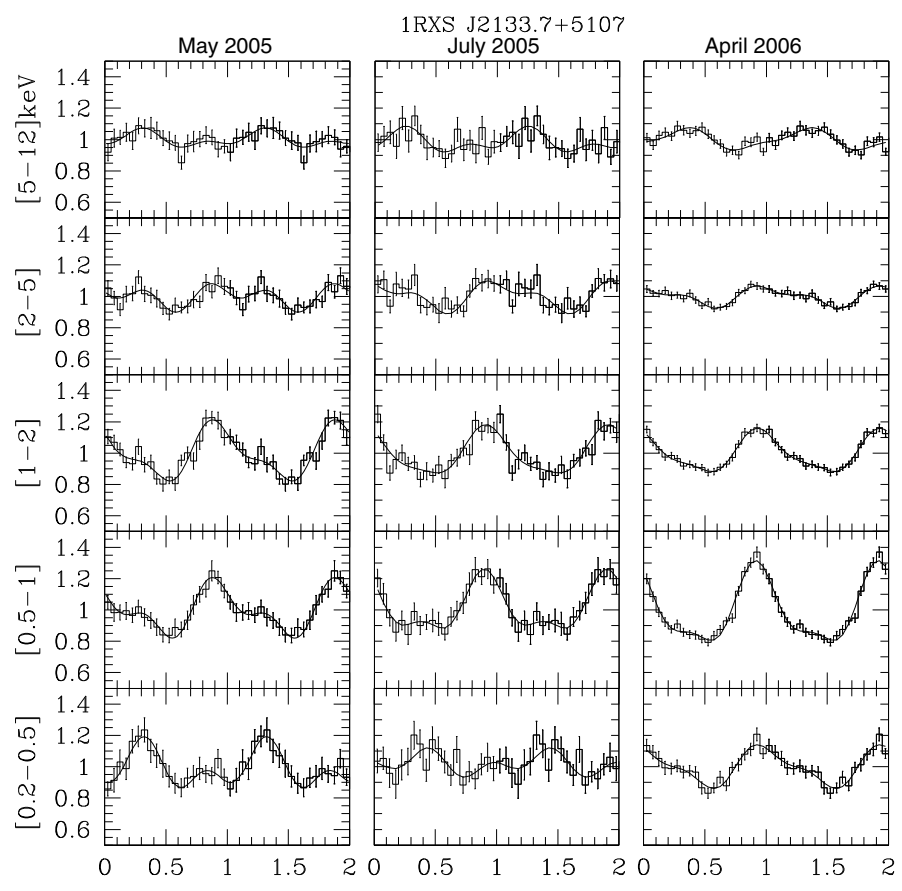

Rotational Phase

Fig. 6. EPIC-pn (May 2005 and July 2005) and XIS spin-folded light curves of RX J2133 extracted in selected energy ranges. All the light curves are folded using the time of maximum quoted in the text.

$\mathrm{S} / \mathrm{N}$ ratio is still good. The average $\mathrm{X}$-ray spectrum is well fitted by a multi-temperature optically thin component plus a rather hot $\left(k T_{\mathrm{BB}} \sim 100 \mathrm{eV}\right)$ blackbody, that is required to best fit the data at energies below $1 \mathrm{keV}$. A total absorber with a low hydrogen column density, likely of interstellar origin (the total Galactic absorption along the direction of the source is $N_{\mathrm{H}, \mathrm{gal}}=7.35 \times 10^{21} \mathrm{~cm}^{-2}$, Dickey \& Lockman (1990)), and a dense $\left(N_{\mathrm{H}} \sim 10^{23} \mathrm{~cm}^{-2}\right)$ absorber partially covering the source are also required. In all the spectral fits we fixed the $N_{\mathrm{H}}$ of the total absorber to the value found for the May 2005 data, since it is not expected to vary with time, and used an unresolved Gaussian line to account for the fluorescence Fe line at $6.4 \mathrm{keV}$. The bestfit parameters are reported in Table 5, where the optically thin components in models $\mathrm{A}-\mathrm{C}$ are the same used in the corresponding spectral models of IGR 0023. We have marked with 1 and 2 the May and July 2005 XMM-Newton spectra, respectively; while 3 indicates the Suzaku spectra ${ }^{2}$. Also for this object, the INTEGRAL spectrum is underpredicted above $\sim 60 \mathrm{keV}$.

In general, we find no significant differences between the spectral parameters of May 2005 and July 2005, with the exception of the normalization of the blackbody component that is slightly higher in the earliest epoch. The absorbed flux in the range $0.2-10.0 \mathrm{keV}$ is $2.4 \times 10^{-11} \mathrm{erg} \mathrm{cm}^{-2} \mathrm{~s}^{-1}$. In the $S u z a k u$ spectra, the temperature of the hottest MEKAL (or the maximum temperature of the CEMEKL model) attains higher values than in 2005. In addition, we find a decrease of the covering fraction of the partial absorber $(50 \%)$ and a lower value of the normalization of the blackbody (with the exception of model $\mathrm{C}$ ). The absorbed flux in the $0.2-10.0 \mathrm{keV}$ range is

\footnotetext{
2 We caution that the quoted errors reflect only the statistical errors. However, our experimentation of adjusting the HXD non X-ray background by $\pm 1 \%$ from the best estimate values did not cause significant changes in the fit parameters, showing that the systematic errors are not dominant in this case.
}
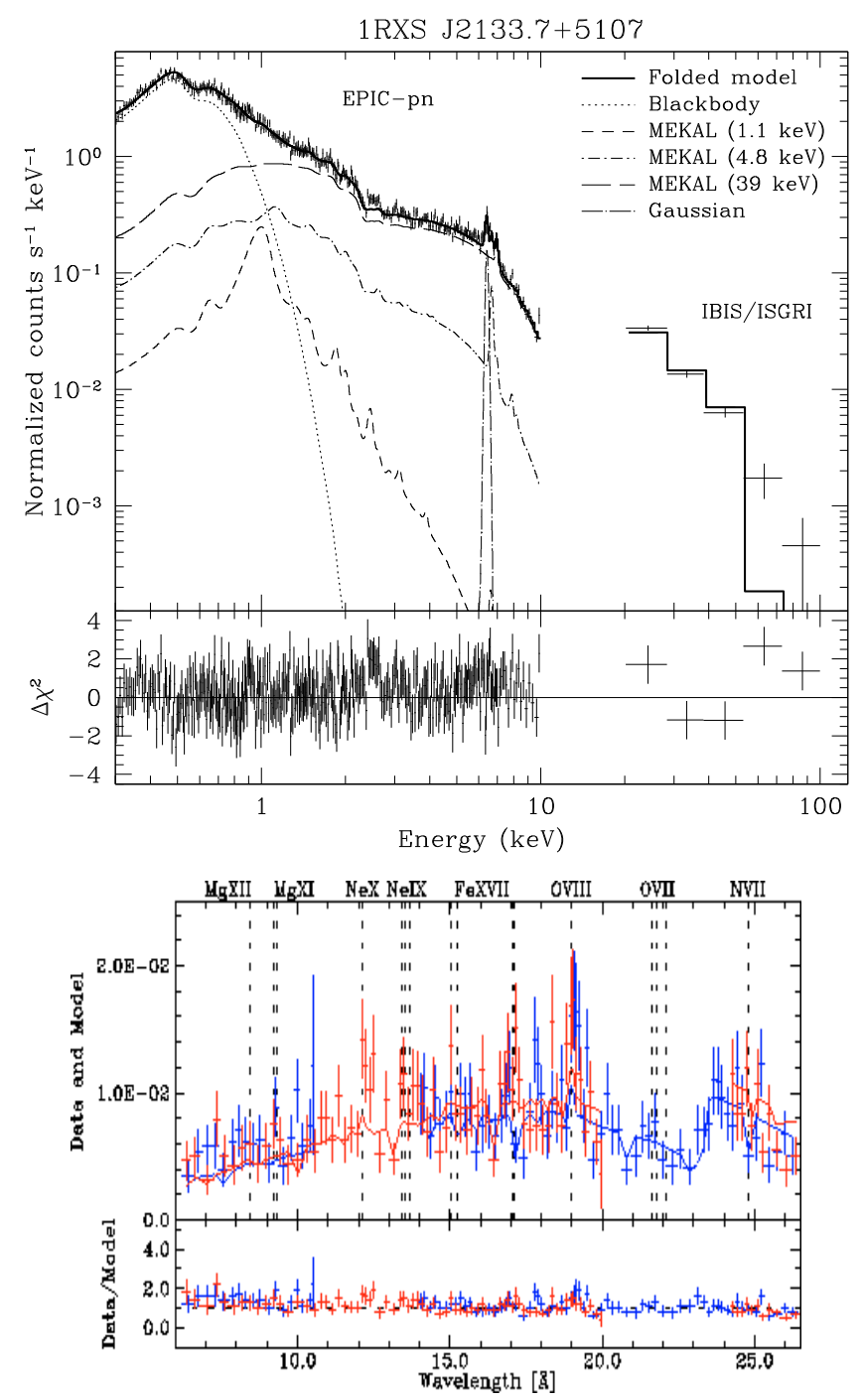

Fig. 7. Upper figure: the EPIC-pn (May 2005) and INTEGRAL spectra of RX J2133 are shown with model C discussed in the text and parameters reported in Table 5 (the combined EPIC-MOS spectrum is not plotted for clarity). The different spectral components contributing to the best-fit model are shown separately. Bottom panel shows the residuals expressed in terms of $\sigma$. Lower figure: the RGS spectra of RX J2133.

$2.5 \times 10^{-11} \mathrm{erg} \mathrm{cm}^{-2} \mathrm{~s}^{-1}$, in agreement with that found in the two observations of 2005. Although we find different values of the bolometric fluxes of both the blackbody and the optically thin components between the two epochs, they are consistent within errors: $F_{\mathrm{BB}}=\left(4.1_{-0.4}^{+0.5}-4.8_{-0.8}^{+0.6}\right) \times 10^{-11} \mathrm{erg} \mathrm{cm}^{-2} \mathrm{~s}^{-1}$ and $F_{\text {hard }}=$ $\left(7.3_{-1.4}^{+1.1}-7.6_{-0.9}^{+0.8}\right) \times 10^{-11} \mathrm{erg} \mathrm{cm}^{-2} \mathrm{~s}^{-1}$ in $2005, F_{\mathrm{BB}}=4.0_{-0.6}^{+1.0} \times$ $10^{-11} \mathrm{erg} \mathrm{cm}^{-2} \mathrm{~s}^{-1}$ and $F_{\text {hard }}>8.3 \times 10^{-11} \mathrm{erg} \mathrm{cm}^{-2} \mathrm{~s}^{-1}$ in 2006 . Therefore, the ratio between the soft and hard X-ray fluxes is of the order of 0.5 .

Because of the similar temperatures of the optically thin components of model $\mathrm{C}$ found at the three epochs, we adopt this model to describe the broad-band spectrum of RX J2133 (see Figs. 7 and 8). Although there is clear evidence of a temperature gradient in the post-shock region, the broad-band spectra do not provide constraints on lower temperatures. Instead, the presence of a low temperature plasma can be inferred from the May 2005 RGS spectra, where the strong O VIII $(0.651 \pm 0.002 \mathrm{keV}, E W=$ $22 \mathrm{eV})$ and $\mathrm{Ne} \mathrm{X}(1.02 \pm 0.01 \mathrm{keV}, E W=14 \mathrm{eV})$ lines are clearly visible (see Fig. 7). The $\mathrm{O}$ VII line at $0.58 \mathrm{keV}(E W=8 \mathrm{eV})$ 
Table 3. Best fit parameters of the phase-resolved spectra of RX J2133.

\begin{tabular}{l|lll|llll}
\hline \hline & \multicolumn{3}{|c|}{ EPIC-pn (May 2005) } & \multicolumn{4}{c}{ XIS (April 2006) } \\
parameters & maximum 1 & maximum 2 & minimum & P1 & P2 & P3 & P4 \\
\hline$N_{\mathrm{H}}^{\text {PCFABS }}\left(10^{23} \mathrm{~cm}^{-2}\right)$ & $1.4_{-0.4}^{+0.6}$ & $0.6 \pm 0.2$ & $1.2_{-0.4}^{+0.6}$ & $1.1 \pm 0.2$ & $1.0 \pm 0.1$ & $1.1 \pm 0.2$ & $1.1 \pm 0.2$ \\
$C_{\mathrm{F}}$ & $0.45_{-0.06}^{+0.05}$ & $0.56_{-0.07}^{+0.06}$ & $0.55_{-0.07}^{+0.05}$ & $0.44 \pm 0.02$ & $0.53 \pm 0.01$ & $0.46 \pm 0.02$ & $0.38 \pm 0.02$ \\
$C_{\mathrm{BB}}\left(10^{-4}\right)$ & $4.7 \pm 0.5$ & $6.4_{-0.9}^{+1.0}$ & $4.6_{-0.6}^{+0.7}$ & $4.7 \pm 0.2$ & $4.9 \pm 0.2$ & $4.2 \pm 0.2$ & $4.4 \pm 0.2$ \\
$C_{1}\left(10^{-4}\right)$ & $14 \pm 3$ & $<2.5$ & $<4.2$ & $<0.8$ & $<0.2$ & $<1.5$ & $6.4 \pm 0.8$ \\
$C_{2}\left(10^{-3}\right)$ & $<2.3$ & $5.2_{-2.6}^{+2.7}$ & $2.6_{-2.2}^{+2.5}$ & $3.6_{-0.7}^{+0.8}$ & $3.9_{-0.6}^{+0.5}$ & $2.7 \pm 0.7$ & $3.7_{-0.7}^{+0.6}$ \\
$C_{3}\left(10^{-2}\right)$ & $1.6 \pm 0.2$ & $1.2 \pm 0.2$ & $1.4 \pm 0.2$ & $1.48_{-0.08}^{+0.09}$ & $1.55_{-0.06}^{+0.07}$ & $1.47_{-0.08}^{+0.09}$ & $1.32 \pm 0.08$ \\
\hline$F_{0.2-10.0 \mathrm{keV}}$ & 2.51 & 2.38 & 2.12 & 2.52 & 2.48 & 2.35 & 2.47 \\
$\left(10^{-11} \mathrm{erg} \mathrm{cm}^{-2} \mathrm{~s}^{-1}\right)$ & & & & & & & 0.80 \\
\hline$\chi_{v}^{2}$ & 1.04 & 0.96 & 0.97 & 0.82 & 0.87 & 0.87 \\
$\left(\chi^{2} /\right.$ d.o.f. $)$ & $(434 / 418)$ & $(266 / 276)$ & $(280 / 290)$ & $(1651 / 2019)$ & $(1721 / 1982)$ & $(1548 / 1939)$ & $(1747 / 2013)$ \\
\hline
\end{tabular}

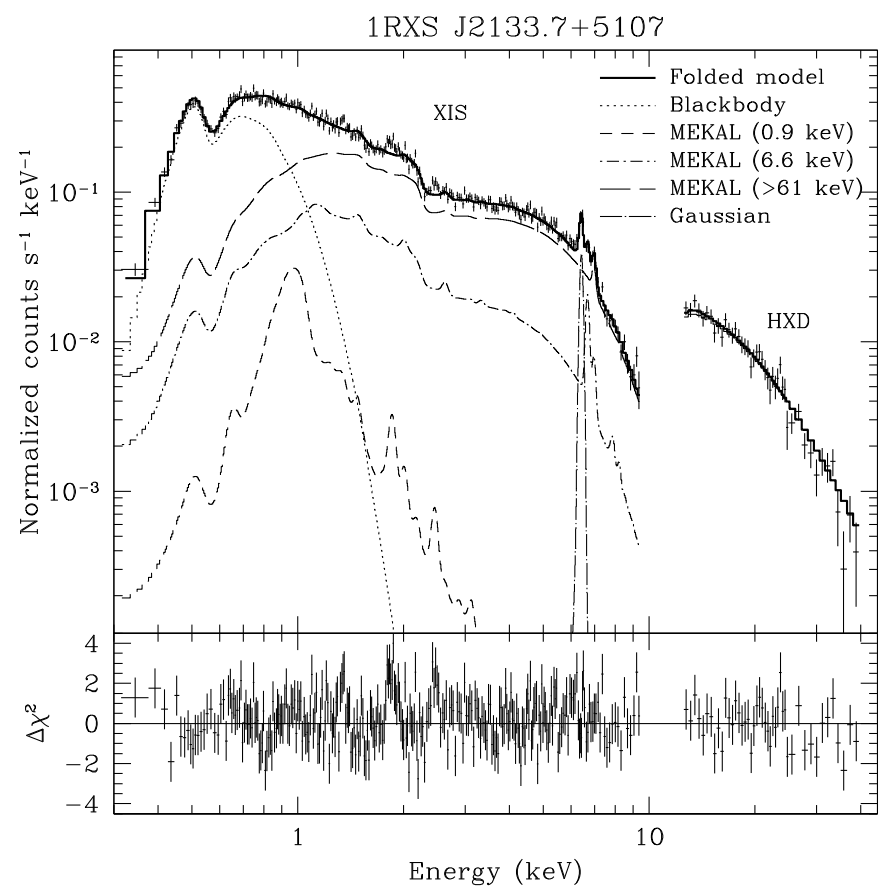

Fig. 8. The Suzaku spectra of RX J2133 are shown with model C discussed in the text and parameters reported in Table 5. The bottom panel shows the residuals.

and the Fe XVII line at $0.73 \mathrm{keV}(E W=11 \mathrm{eV})$ are likely present, while there is only a weak evidence of the Ne IX line. We then estimate a ratio $\sim 2.5$ between the $\mathrm{H}-$ and He-like oxygen lines, that would imply a temperature of $\sim 0.3-0.4 \mathrm{keV}$. This is also supported by the $\mathrm{Ne}$ line ratio of $\sim 1.5$, again indicating $k T \sim 0.4 \mathrm{keV}$.

The Fe line at $6.4 \mathrm{keV}$ has a large equivalent width $(E W=$ 150-170 eV), suggesting a Compton reflection component. However, we do not find improvements of the fits with the inclusion of a reflection component. We then applied the PSR model to the combined XMM-Newton (May 2005) and INTEGRAL spectra $\left(\chi^{2} /\right.$ d.o.f. $\left.=347 / 279\right)$, as well as to the Suzaku spectra $\left(\chi^{2} /\right.$ d.o.f. $\left.=395 / 359\right)$. We find a shock temperature of $50 \pm 2 \mathrm{keV}$ and $53 \pm 3 \mathrm{keV}$, respectively, while the parameters of the absorbers and of the blackbody component are consistent, within errors, with those found using models A-C.

We also performed a phase-resolved analysis of the EPIC-pn (May 2005) and XIS spectra. The former was extracted in phase intervals approximately centered on the two maxima $(\phi=0.72-0.94$ and $\phi=0.16-0.28)$ and at the minimum $(\phi=0.44-0.61)$. Instead, the higher quality XIS spectrum was extracted in 4 consecutive phase intervals, namely $\phi=$ 0.05-0.3 (P1), 0.3-0.55 (P2), 0.55-0.8 (P3) and 0.8-1.05 (P4). We fitted these spectra adopting model C, fixing $N_{\mathrm{H}}$ of the total absorber, the temperatures of all the emission components and $A_{Z}$ to the values found for the corresponding average spectrum. The results reported in Table 3 show significant differences in some of the parameters. The normalization of the soft MEKAL increases at the primary maximum in the two epochs, whilst only upper limits are found at other phases. Also, a decrease of the hydrogen column density of the partial absorber and a slight increase of the blackbody normalization at the secondary maximum are found only in May 2005.

\section{Discussion}

\subsection{IGR 0023}

The XMM-Newton data confirm the previously identified optical $561 \mathrm{~s}$ period as the spin period of the accreting WD. A pseudoperiodicity of the order of $1 \mathrm{~h}$, though with a significance level below $3 \sigma$, seems to be present above $1 \mathrm{keV}$ and was previously found also in optical data (Bonnet-Bidaud et al. 2007). This variability, if real, is not easy to explain. Assuming a circular Keplerian orbit, a periodicity of $1.09 \mathrm{~h}$ implies a radius of $\sim 3 \times$ $10^{10} \mathrm{~cm}$. For comparison, adopting a mass ratio $q=0.5$ and a WD mass of $\sim 0.9 M_{\odot}$ (see below), the outer edge of the accretion disk would be $4 \times 10^{10} \mathrm{~cm}$. This may suggest an origin within the disk, but unlikely due to absorption effects as instead found in many IPs (Parker et al. 2005).

The spin pulse is detected only below $2 \mathrm{keV}$, where the power spectrum shows substantial signal also at $2 \omega$. The doublehumped spin pulse and the increase of amplitudes with decreasing energy could be the signatures of two accreting poles, as well as of variable absorption. From the phase resolved spectra, we find a decrease by a factor $\sim 2$ in the normalization constant of the MEKAL at $0.17 \mathrm{keV}$, as well as an increase of a dense partial absorber at the secondary maximum. This prevents us to isolate the true contribution from a secondary pole. In addition, the optical pulse is single-humped, thus not revealing a secondary pole. Hence, we are left with the uncertainty on whether the X-ray pulse shape is due to the presence of a secondary and less active pole or to phase-dependent absorption effects in the accretion curtain (Rosen et al. 1988).

The X-ray emission in IGR 0023, extending up to $\sim 90 \mathrm{keV}$, requires a multi-temperature optically thin plasma with a 
temperature distribution more complex than a simple power law. The spectral fits indicate a likely maximum temperature of $\sim 50 \mathrm{keV}$. High plasma temperatures were also inferred from broad-band analysis of the combined XMM-Newton INTEGRAL spectra of 1RXS J173021.5-055933 (de Martino et al. 2008) and from hard X-ray observations of several other IPs (Landi et al. 2009; Suleimanov et al. 2008; Brunschweiger et al. 2009). Although caution has to be taken to interpret these temperatures in terms of the WD mass, the extension of spectra above $30 \mathrm{keV}$ allow us to directly observe the exponential cut-off of the underlying continuum and hence the inferred temperatures are likely to be more reliable than those based only on softer energy coverages (e.g. XMM-Newton). For IGR 0023 we estimate a WD mass of $0.91_{-0.16}^{+0.14} M_{\odot}$, consistent with that found by Brunschweiger et al. (2009) from the analysis of Swift/BAT data. Although relatively massive WD primaries were inferred in several magnetic CVs, the mass distribution of magnetic CV primaries is not very much different from that of non-magnetic systems (Ramsay 2000). Hence, for a CV to be a hard X-ray source, there should be an additional parameter that plays an important role.

The hard X-ray bolometric flux $F_{\text {hard }}=1.8 \times$ $10^{-11} \mathrm{erg} \mathrm{cm}^{-2} \mathrm{~s}^{-1}$ is an approximate estimate of the total accretion luminosity, as the pulsed components at other wavelengths need to be included as well as a secure distance estimate is missing. Here we can obtain only a lower limit using the $8 \%$ pulsed $V$ band contribution, adopting $E(B-V)=0.35$ as derived by the $N_{\mathrm{H}}$ of the total absorber (Ryter et al. 1975). Also, assuming a lower limit of $500 \mathrm{pc}$ for the distance of IGR 0023 , as estimated by Bonnet-Bidaud et al. (2007) using the 2MASS $K$ band magnitude when attributed solely to the secondary star (Knigge 2006), we obtain $L_{\text {accr }}>L_{\text {hard }}+L_{V}=5.5 \times$ $10^{32} d_{500 \mathrm{pc}}^{2} \mathrm{erg} \mathrm{s}^{-1}$. This in turn gives us a lower limit for the accretion rate $\dot{M}>2.5 \times 10^{15} d_{500 \mathrm{pc}}^{2} \mathrm{~g} \mathrm{~s}^{-1}$. Unless the distance is much larger and the hard X-rays do not trace the bulk of accretion, this value is much lower than $2.2 \times 10^{17} \mathrm{~g} \mathrm{~s}^{-1}$ predicted for its $4.033 \mathrm{~h}$ orbital period (Warner 1995).

The short spin periods of IGR 0023 and RX J2133 are similar to those of YY Dra (Patterson et al. 1992), V405 Aur (Skillman 1996) and 1RXS J070407.9+262501 (Gänsicke et al. 2005). Fast rotators were proposed to possess weakly magnetized WDs (Norton et al. 1999), although V405 Aur and RX J2133 are among the few IPs showing polarized optical emissions. Assuming a pure disk accretion, as indicated by the absence of orbital sidebands in the power spectrum, a lower limit for the magnetic moment $\mu \gtrsim 9 \times 10^{31} d_{500 \mathrm{pc}} \mathrm{G} \mathrm{cm}^{3}$ is obtained using the inferred values of $M_{\mathrm{WD}}$ and $\dot{M}$. This could suggest that IGR 0023 is not strongly magnetized and not spinning at equilibrium.

\section{2. $R X J 2133$}

Our X-ray analysis confirms the $571 \mathrm{~s}$ period detected in optical photometry and polarimetry (Bonnet-Bidaud et al. 2006; Katajainen et al. 2007) as the rotational period of the WD primary. RX J2133 is, therefore, one of the most asynchronous systems among IPs, with a ratio between the spin and the orbital periods of 0.022 .

The double peaked X-ray pulse shows a complex energy dependence and might be interpreted in terms of absorption and two emitting poles. The energy-resolved light curves suggest that the primary pole dominates the emission in the energy range $0.5-5.0 \mathrm{keV}$, while the two poles contribute similarly at higher energies. This is also found in the phase-resolved spectral analysis where the main changes are due to the emitting volume of an intermediate temperature region. The soft X-ray blackbody component is seen at all spin phases, suggesting either that the irradiated main pole is never occulted or that reprocessing occurs at both poles. However, in 2005 the secondary maximum is dominant in the soft band, and at this phase we find a decrease in the absorption and a slight increase in the blackbody normalization. Hence, when the main pole points away from the observer, we are viewing the contribution of the irradiated area of the secondary pole. In order to have a lower absorption at this phase, a modification of the standard accretion curtain model (Norton et al. 1999), where the optical depth is largest when viewing the curtain perpendicularly, could be envisaged.

UV and optical data also support a scenario with two accreting poles whose contributions are however variable with time. The XMM-Newton UV observation of July 2005 shows a weak modulation antiphased with the X-ray pulse, while in May 2005 the UV light is not modulated but the $B$ band pulse is single-peaked. Previous white light photometry acquired in 2003 (Bonnet-Bidaud et al. 2006) shows the presence of two poles, being the first harmonic of both the spin frequency and the beat clearly detected. This is not seen in the 2006 optical photometry and polarimetry (Katajainen et al. 2007) as well as in the Suzaku X-ray data in the same year. Note that Bonnet-Bidaud et al. (2006) suggested a relatively low binary inclination $\left(i \leq 45^{\circ}\right)$ and the behavior of the circular polarization along the spin cycle indicates a magnetic colatitude $\beta \sim 90^{\circ}-i$ (Katajainen et al. 2007). This implies $i \geq 45^{\circ}$ and hence that the secondary pole, whenever active, can be observed.

The broad-band X-ray spectrum of RX J2133 is well fitted by a multi-temperature optically thin plasma with a likely maximum temperature $k T_{\max } \sim 50 \mathrm{keV}$. As for IGR 0023, a power-law multi-temperature flow appears a too simple and inadequate description of the post-shock emitting region, implying that the emergent spectrum is highly sensitive to local pressure and temperature across the flow. Furthermore, a blackbody component at $100 \mathrm{eV}$ is also required to account for the soft $\mathrm{X}$-ray emission. To date this component has been detected in $\sim 42 \%$ of the IPs, thus appearing a common characteristics of magnetic CVs and not solely of polars (Anzolin et al. 2008). If produced by the heating of a small area surrounding the WD polar cap, the range of values of the normalization constant obtained by applying model C to the spectra of RX J2133 (see Table 5) implies an emission area of $1.6-1.9 \times 10^{13} d_{600 \mathrm{pc}}^{2} \mathrm{~cm}^{2}$. Here we use a minimum distance to the source as derived by Bonnet-Bidaud et al. (2006) using 2MASS $K$ band magnitude. Assuming $k T_{\max } \sim k T_{\text {shock }}$, we infer a WD mass of $0.93 \pm$ $0.04 M_{\odot}$, in agreement with the values found by Bonnet-Bidaud et al. (2006) and Brunschweiger et al. (2009), and a radius of $5.6 \times 10^{8} \mathrm{~cm}$ (Nauenberg 1972). The fractional area of the blackbody emitting region is $f \sim 2-3 \times 10^{-6} d_{600 \mathrm{pc}}^{2}$. This could represent a small core onto the WD pole, as found in other soft X-ray IPs with similar temperatures (Haberl et al. 2002; de Martino et al. 2008). We also point out that a $\sim 100 \mathrm{eV}$ blackbody would imply that the emission from the accretion region locally exceeds the Eddington limit. At that temperature, the radiation pressure is 4 times stronger than the gravity at the surface of a $0.93 M_{\odot}$ WD. Clearly we still need a better understanding of emission mechanisms to solve this puzzle.

The bolometric flux of the blackbody represents $\sim 40 \%$ of the total bolometric X-ray emission. RX J2133 shows circularly polarized emission up to $\sim 3 \%$ and hence the flux due to 

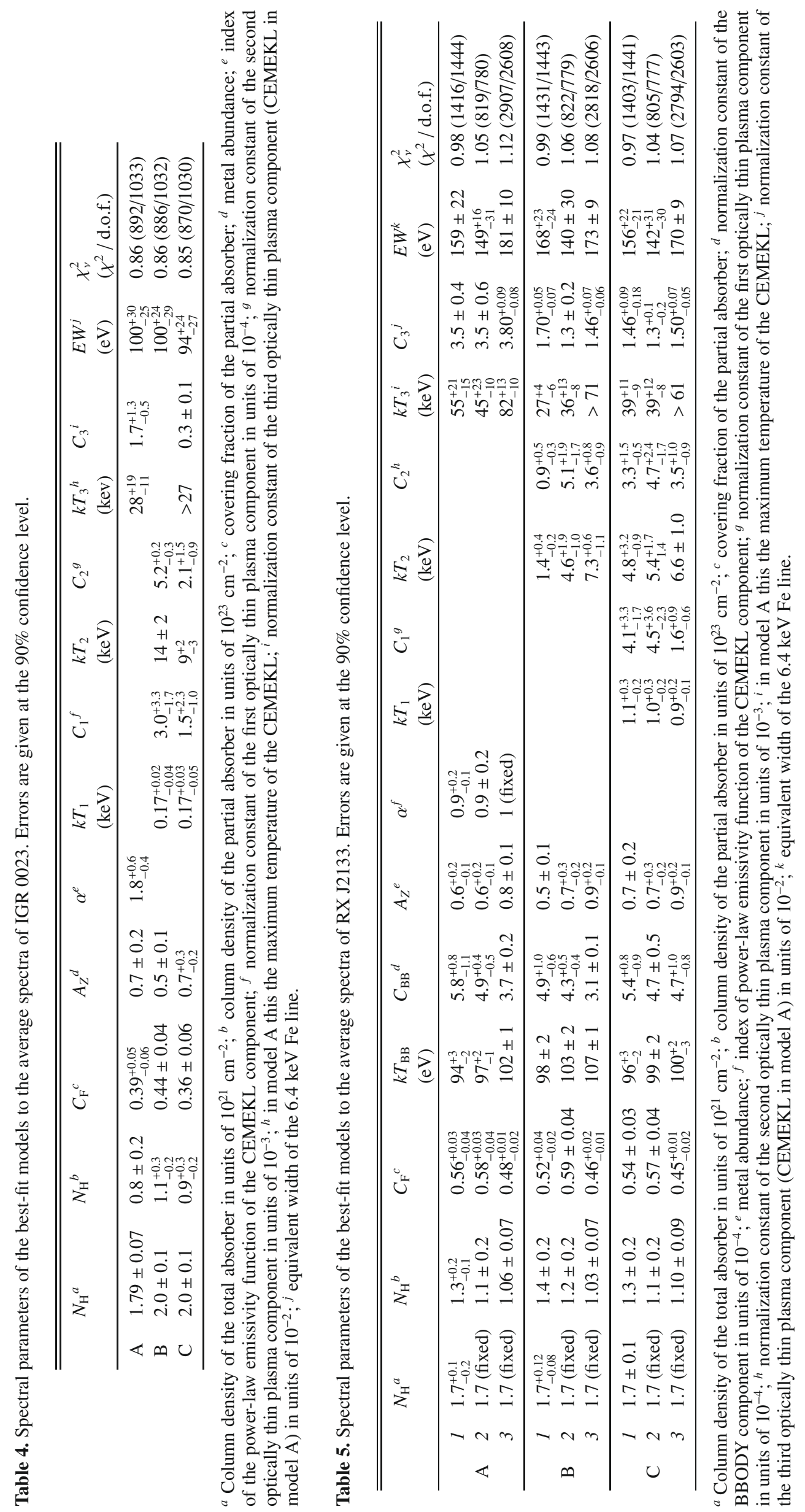
cyclotron radiation cannot be neglected. Since the blackbody flux does not exceed that of the hard X-ray emission, the reprocessed radiation seen in the soft X-rays does not balance the primary radiation components. We have found that the UV emission is modulated by $6 \%$ at the spin period in July 2005, but antiphased with respect to the hard X-ray component, thus suggesting that the reprocessed radiation at the WD surface is also emitted at UV wavelengths. The pulsed UV flux observed in July 2005 , dereddened for $E(B-V)=0.25$, is $F_{U V M 2} \sim$ $2.1 \times 10^{-15} \mathrm{erg} \mathrm{cm}^{-2} \mathrm{~s}^{-1} \AA^{-1}$. This value is much larger than that expected from the extrapolation of the soft X-ray flux towards UV wavelengths. Also, the UV flux integrated over the $U V M 2$ band is only $4 \%$ of the soft X-ray bolometric flux and hence only provide a lower limit to the UV luminosity.

To evaluate the mass accretion rate we then consider the hard and soft X-ray components, as well as the modulated fraction of the flux in the $B$ band of the May 2005 observation. A lower limit for the accretion luminosity is therefore: $L_{\text {accr }} \gtrsim 5 \times$ $10^{33} d_{600 \mathrm{pc}}^{2} \operatorname{erg~s}^{-1}$. We obtain $\dot{M} \gtrsim 2.3 \times 10^{16} d_{600 \mathrm{pc}}^{2} \mathrm{~g} \mathrm{~s}^{-1}$, much lower than the secular value of $1.9 \times 10^{18} \mathrm{~g} \mathrm{~s}^{-1}$ predicted for its long $7.2 \mathrm{~h}$ orbital period. From the detection of circular polarization in the optical range, that peaks in the $V$ band, Katajainen et al. (2007) suggest a magnetic moment $\mu$ in the range $3 \times 10^{33}-3 \times 10^{34}$. The condition for accretion would then imply $\dot{M} \gtrsim 5.3 \times 10^{17} \mathrm{~g} \mathrm{~s}^{-1}$. Although this value is probably at the high side, it might indicate that the a substantial contribution to the accretion luminosity comes from cyclotron radiation and not from the hard X-rays.

Because of the wide range of temperatures found for the soft X-ray component, Anzolin et al. (2008) proposed that $T_{\mathrm{BB}}$ could be related to the magnetic field strength. Lower temperatures would indicate higher field systems because of larger irradiated WD areas by cyclotron radiation. However, the recent detection of significant polarized emission in RX J2133 (Katajainen et al. 2007) and 1RXS J173021.5-055933 (Butters et al. 2009) adds them to the subset of soft X-ray and polarized IPs together with V2400 Oph, PQ Gem and V405 Aur. While the degree of polarization could also be affected by the accretion geometry, these five polarized systems possess soft blackbody components that span a wide range of temperatures, with three out of five displaying hot blackbodies. The inferred magnetic field strengths are quite uncertain for most systems, being 9-21 MG for PQ Gem (Potter et al. 1997), 9-27 MG for V2400 Oph (Vaeth 1997) and 30 MG for V405 Aur (Piirola et al. 2008). A 20 MG field was proposed for RX J2133 (Katajainen et al. 2007), while no field estimate is given for 1RXS J173021.5-055933 (Butters et al. 2009). To really test the proposal made by Anzolin et al. (2008), spectro-polarimetric measures of the magnetic field strengths are essential for all soft systems. Furthermore, there are indications that the reprocessed radiation emerges in the UV range also in IPs, as found in PQ Gem (Stavroyiannopoulos et al. 1997) and UU Col (de Martino et al. 2006). This aspect is essential to properly determine the reprocessed energy budget.

\section{Conclusions}

The two CVs IGR 0023 and RX J2133 have been confirmed as true members of the IP class by using pointed X-ray observations with XMM-Newton and Suzaku satellites. A strong pulsation at the WD rotational period dominates the power spectra of IGR 0023 below $2 \mathrm{keV}$, while for RX J2133 it is detected up to $12 \mathrm{keV}$. Both systems have fast rotating WDs, with $P_{\omega}$ of $561 \mathrm{~s}$ for IGR 0023 and $571 \mathrm{~s}$ for RX J2133. The latter is one of the most asynchronous systems among IPs, having a ratio between the spin and the orbital periods of 0.022 . The fast rotation and very long orbital period, together with the detection of substantial polarized emission, suggests that RX J2133 is likely a young IP that will evolve into a polar when attaining synchronism.

Their broad-band X-ray spectra were analyzed including also INTEGRAL data, that allowed us to cover the energy range $0.2-100 \mathrm{keV}$. These are well described by a multi-temperature plasma emission, with a minimum temperature of $0.2-0.3 \mathrm{keV}$ and a maximum temperature of $\sim 50 \mathrm{keV}$, which implies relatively massive WDs. While this might suggest that hard X-ray CVs harbor massive primaries, it has still to be understood if this is the only ingredient for a $\mathrm{CV}$ to be a hard X-ray source.

In RX J2133, a $\sim 100 \mathrm{eV}$ blackbody emission is also required to fit the soft portion of the spectrum. The temperature of this component is similar to that found in V2400 Oph (de Martino et al. 2004) and 1RXS J173021.5-055933 (de Martino et al. 2008), both of them also showing circularly polarized radiation. It is however different from that found in the other two soft X-ray and polarized IPs, PQ Gem and V405 Aur (de Martino et al. 2004; Anzolin et al. 2008). This casts doubts on the possible relation between the soft X-ray temperature and the magnetic field strength proposed by Anzolin et al. (2008). The present work also opens a further question on whether the reprocessed radiation in the soft and polarized IPs also emerges at UV wavelengths, as recently demonstrated by König et al. (2006) for the polar prototype AM Her.

Furthermore, the fact that RX J2133, V2400 Oph and 1RXS J173021.5-055933 are bright hard X-ray sources and are also polarized IPs might not imply that cyclotron cooling decreases the hard X-ray flux (Woelk \& Beuermann 1996; Fischer \& Beuermann 2001), at least for the magnetic strength values covered by these IPs. Observations aiming at measuring the magnetic field strength of IPs will help in making a clear picture of emission mechanisms in these systems.

Acknowledgements. We acknowledge the XMM-Newton MSSL and SOC staff for help in the reduction of the OM data. D.d.M. and G.A. acknowledge financial support from ASI under contract ASI/INAF I/023/05/06 and ASI/INAF I/088/06/0 and from INAF under contract PRIN-INAF 2007 N.17. We also acknowledge useful suggestions and comments from the referee, Prof. Klaus Beuermann, that helped us to improve this work.

\section{References}

Aizu, K. 1973, Prog. Theor. Phys., 49, 1184

Anzolin, G., de Martino, D., Bonnet-Bidaud, J.-M., et al. 2008, A\&A, 489, 1243

Barlow, E. J., Knigge, C., Bird, A. J., et al. 2006, MNRAS, 372, 224

Bikmaev, I. F., Revnivtsev, M. G., Burenin, R. A., \& Sunyaev, R. A. 2006, Astron. Lett., 32, 588

Bird, A. J., Malizia, A., Bazzano, A., et al. 2007, ApJS, 170, 175

Bonnet-Bidaud, J. M., Mouchet, M., de Martino, D., Silvotti, R., \& Motch, C. 2006, A\&A, 445, 1037

Bonnet-Bidaud, J. M., de Martino, D., Falanga, M., Mouchet, M., \& Masetti, N. 2007, A\&A, 473, 185

Brunschweiger, J., Greiner, J., Ajello, M., \& Osborne, J. 2009, A\&A, 496, 121

Butters, O. W., Katajainen, S., Norton, A. J., Lehto, H. J., \& Piirola, V. 2009, A\&A, 496, 891

de Martino, D., Matt, G., Belloni, T., Haberl, F., \& Mukai, K. 2004, A\&A, 415, 1009

de Martino, D., Matt, G., Mukai, K., et al. 2006, A\&A, 454, 287

de Martino, D., Matt, G., Mukai, K., et al. 2008, A\&A, 481, 149

den Hartog, P. R., Hermsen, W., Kuiper, L., et al. 2006, A\&A, 451, 587

den Herder, J. W., Brinkman, A. C., Kahn, S. M., et al. 2001, A\&A, 365, L7

Dickey, J. M., \& Lockman, F. J. 1990, ARA\&A, 28, 215

Fischer, A., \& Beuermann, K. 2001, A\&A, 373, 211

Fukazawa, Y., Mizuno, T., Watanabe, S., et al. 2009, PASJ, in press

Gänsicke, B. T., Marsh, T. R., Edge, A., et al. 2005, MNRAS, 361, 141 
Goldwurm, A., David, P., Foschini, L., et al. 2003, A\&A, 411, L223

Haberl, F., Motch, C., \& Zickgraf, F.-J. 2002, A\&A, 387, 201

Jansen, F., Lumb, D., Altieri, B., et al. 2001, A\&A, 365, L1

Katajainen, S., Butters, O. W., Norton, A. J., Lehto, H. J., \& Piirola, V. 2007, A\&A, 475, 1011

Knigge, C. 2006, MNRAS, 373, 484

König, M., Beuermann, K., \& Gänsicke, B. T. 2006, A\&A, 449, 1129

Koyama, K., Tsunemi, H., Dotani, T., et al. 2007, PASJ, 59, S23

Landi, R., Bassani, L., Dean, A. J., et al. 2009, MNRAS, 392, 630

Lebrun, F., Leray, J. P., Lavocat, P., et al. 2003, A\&A, 411, L141

Masetti, N., Bassani, L., Bazzano, A., et al. 2006, A\&A, 455, 11

Masetti, N., Mason, E., Morelli, L., et al. 2008, A\&A, 482, 113

Mason, K. O., Breeveld, A., Much, R., et al. 2001, A\&A, 365, L36

Mitsuda, K., Bautz, M., Inoue, H., et al. 2007, PASJ, 59, 1

Motch, C., Guillout, P., Haberl, F., et al. 1998, A\&AS, 132, 341

Muno, M. P., Baganoff, F. K., Bautz, M. W., et al. 2004, ApJ, 613, 326

Nauenberg, M. 1972, ApJ, 175, 417

Norton, A. J., Beardmore, A. P., Allan, A., \& Hellier, C. 1999, A\&A, 347, 203

Parker, T. L., Norton, A. J., \& Mukai, K. 2005, A\&A, 439, 213

Patterson, J., Schwartz, D. A., Pye, J. P., et al. 1992, ApJ, 392, 233

Piirola, V., Vornanen, T., Berdyugin, A., \& Coyne, S. J., G. V. 2008, ApJ, 684, 558

Potter, S. B., Cropper, M., Mason, K. O., Hough, J. H., \& Bailey, J. A. 1997, MNRAS, 285, 82
Ramsay, G. 2000, MNRAS, 314, 403

Revnivtsev, M., Sazonov, S., Krivonos, R., Ritter, H., \& Sunyaev, R. 2008, A\&A, 489, 1121

Rosen, S. R., Mason, K. O., \& Cordova, F. A. 1988, MNRAS, 231, 549

Ryter, C., Cesarsky, C. J., \& Audouze, J. 1975, ApJ, 198, 103

Sazonov, S., Revnivtsev, M., Gilfanov, M., Churazov, E., \& Sunyaev, R. 2006, A\&A, 450, 117

Schenker, K., Wynn, G. A., \& Speith, R. 2004, in Magnetic Cataclysmic Variables, ed. S. Vrielmann, \& M. Cropper, IAU Colloq., 190, ASP Conf. Ser., 315,8

Skillman, D. R. 1996, PASP, 108, 130

Stavroyiannopoulos, D., Rosen, S. R., Watson, M. G., Mason, K. O., \& Howell, S. B. 1997, MNRAS, 288, 891

Strüder, L., Briel, U., Dennerl, K., et al. 2001, A\&A, 365, L18

Suleimanov, V., Poutanen, J., Falanga, M., \& Werner, K. 2008, A\&A, 491, 525

Takahashi, T., Abe, K., Endo, M., et al. 2007, PASJ, 59, S35

Turner, M. J. L., Abbey, A., Arnaud, M., et al. 2001, A\&A, 365, L27

Ubertini, P., Lebrun, F., Di Cocco, G., et al. 2003, A\&A, 411, L131

Vaeth, H. 1997, A\&A, 317, 476

Warner, B. 1995, Cataclysmic variable stars (Cambridge: Cambridge University Press)

Winkler, C., Courvoisier, T. J.-L., Di Cocco, G., et al. 2003, A\&A, 411, L1

Woelk, U., \& Beuermann, K. 1996, A\&A, 306, 232

Wu, K., Chanmugam, G., \& Shaviv, G. 1994, ApJ, 426, 664 